\title{
Carbon removal from trenches on EUV reticles
}

\author{
N.B. Koster ${ }^{a^{*}}$, C.P.E.C. Geluk ${ }^{a}$, T.W. Versloot ${ }^{b}$, J.P.B. Janssen ${ }^{a}$, Y. Fleming ${ }^{c}$, T. Wirtz ${ }^{c}$ \\ ${ }^{a}$ TNO, Stieltjesweg 1, 2628 CK Delft, The Netherlands; ${ }^{b} E S A$, Keplerlaan 1, Noordwijk, The \\ Netherlands; ${ }^{\mathrm{c}}$ Department "Science and Analysis of Materials" (SAM), Centre de Recherche Public \\ - Gabriel Lippmann, 41 rue du Brill, L-4422 Belvaux, Luxembourg
}

\begin{abstract}
We report on our investigation of dry cleaning of reticles with a microwave induced hydrogen plasma on dummy reticles. The dummy reticles were manufactured with $70 \mathrm{~nm}$ ALD grown TaN on a Ru surface and test structures were patterned with lines and spaces ranging between 250 and $400 \mathrm{~nm}$. After processing the test structures were contaminated with e-beam grown carbon and exposed in our plasma facility to remove the carbon with the aid of a hydrogen plasma. Analysis of the samples was performed with SEM/EDX and with SIMS-SPM to verify the complete removal of carbon from the bottom of the trench. Analysis showed that there are small traces of carbon still present on the samples. This can be contributed to contamination which has occurred during transport and storage or that the grown carbon has some edges which are higher due to localized high intensity in the focus of the e-beam.
\end{abstract}

Keywords: EUV reticles, cleaning, plasma, SIMS-SPM

\section{INTRODUCTION}

With the insertion of EUV HVM production being pushed back towards the 10 or $7 \mathrm{~nm}$ node, the feature size on reticles is also decreasing towards less than $30 \mathrm{~nm}$. Besides the increasing risk of particles becoming a killer defect at these critical dimensions, the contribution of EUV induced carbon in the trenches can seriously affect the optical performance of the reticle. For the particles contamination the discussion on the possibility of a pellicle has recently been started again as a possible protective means, however this will not solve the carbon contamination problem. With EUV powers needed for the HVM production of the $\mathrm{X} \mathrm{nm}$ node growing towards $1 \mathrm{~kW}$, the growth of carbon will also increase. Even with a pellicle, residual hydrocarbons will be present in between the space of the reticle and pellicle. Although wet cleaning has been reported as successful, it can be questioned if the liquid can enter to the bottom of the trench to remove the carbon when he size of the trench approaches $30 \mathrm{~nm}$ with an aspect ratio of 3 to 5 . An overview of a number of possible cleaning techniques has been described by L. Scaccaborozzi et $\mathrm{al}^{[1]}$, although alternatives have not been fully examined. With a pellicle mounted on the reticle, wet cleaning is impossible and either the pellicle has to be removed or a dry technique is necessary. For a cleaning technique with the pellicle still on the reticle the active species have to be flushed in the space between reticle and pellicle and the waste of the cleaning process has to be gaseous in order to be removed. Plasma cleaning is a technique where the active and waste products are gaseous and depending on the active species $\left(\mathrm{O}_{2}\right.$ or $\mathrm{H}_{2}$ ) the carbon is converted in either $\mathrm{CO}_{2}$ or $\mathrm{CH}_{4}$ which are both gasses and can be pumped away.

We have examined the removal of carbon in trenches of dummy reticles using our Shielded Microwave Induced Remote Plasma (SMIRP) with $\mathrm{H}_{2}$ as active gas. In our previous work, we already have shown that EUV mirrors and reticles can be cleaned without any damage or loss of reflectivity ${ }^{[2]}$.

\section{EXPERIMENTAL}

\subsection{Sample manufacturing}

As the availability of EUV reticles, which can be cut into pieces for analysis, is very scarce we made our own dummy structures on $\mathrm{Si}$ wafers. The test structure consists of dense lines and spaces arranged in a square as shown in Figure $\mathbf{1}$. One test structure consists of four smaller squares with different spacings, for this case we used 250, 300, 350 and 400 $\mathrm{nm}$ lines and spaces. Although this is not the dense spacing as can be expected on a real reticle we had to limit to these

*Norbert.koster@tno.nl; phone +31888666339; www.tno.nl

Photomask Technology 2014, edited by Paul W. Ackmann, Naoya Hayashi, Proc. of SPIE Vol. 9235, $923517 \cdot$ ? 2014 SPIE · CCC code: 0277-786X/14/\$18 · doi: 10.1117/12.2066471 
sizes otherwise it would not have been possible to do the SIM-SPM analysis in the bottom of the trench due to the AFM tip size, but smaller dimensions down to $90 \mathrm{~nm}$ have also been fabricated. A $50 \mathrm{~nm}$ thick Ru layer was sputter coated on the wafers and checked for layer thickness and uniformity. Next a TaN layer with an approximate layer thickness of 70 $\mathrm{nm}$ was deposited using Atomic Layer Deposition (ALD) and $200 \mathrm{~nm}$ of ZEP520 resist was spin coated on the wafer.

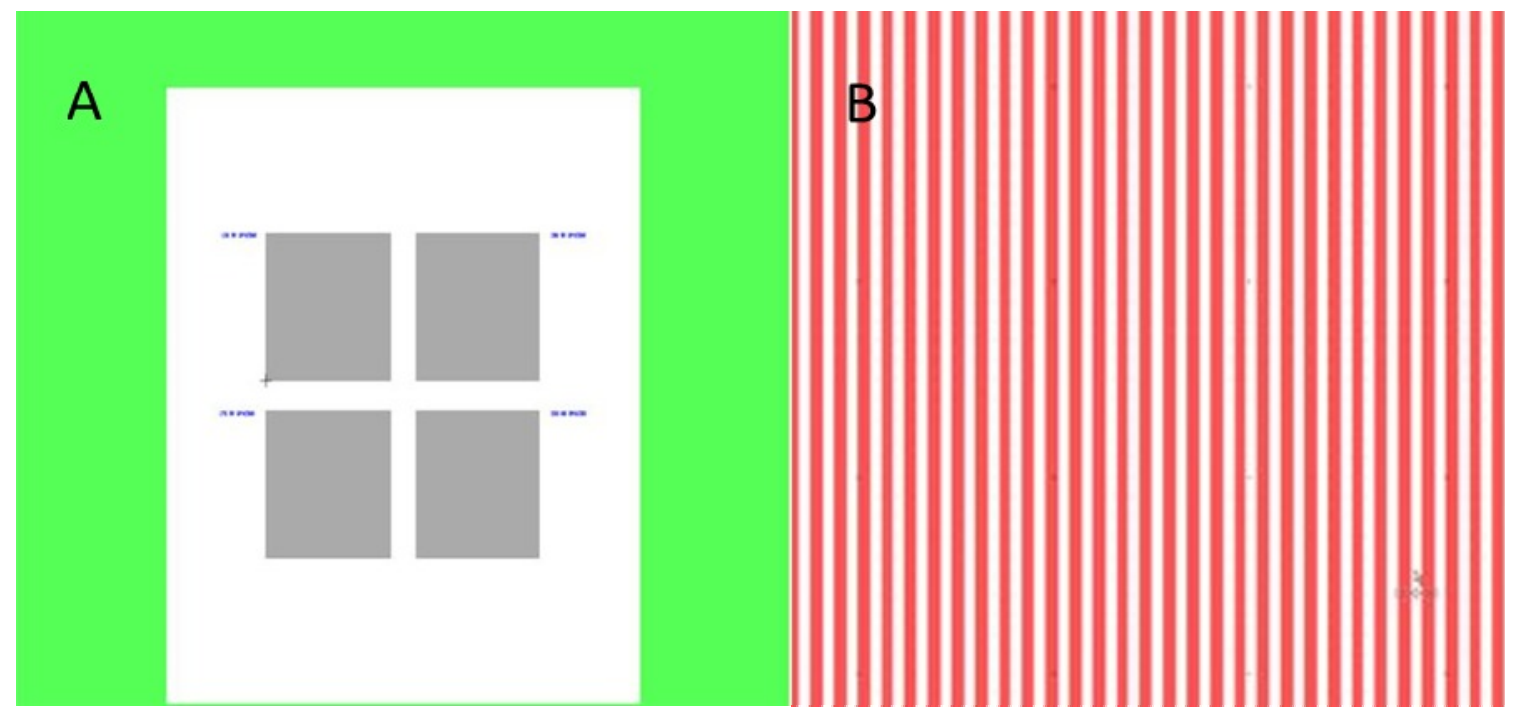

Figure 1: GDS drawing of the test structure, with a) full structure showing the four squares filled with lines and spaces and b) single structure with the lines and spaces.

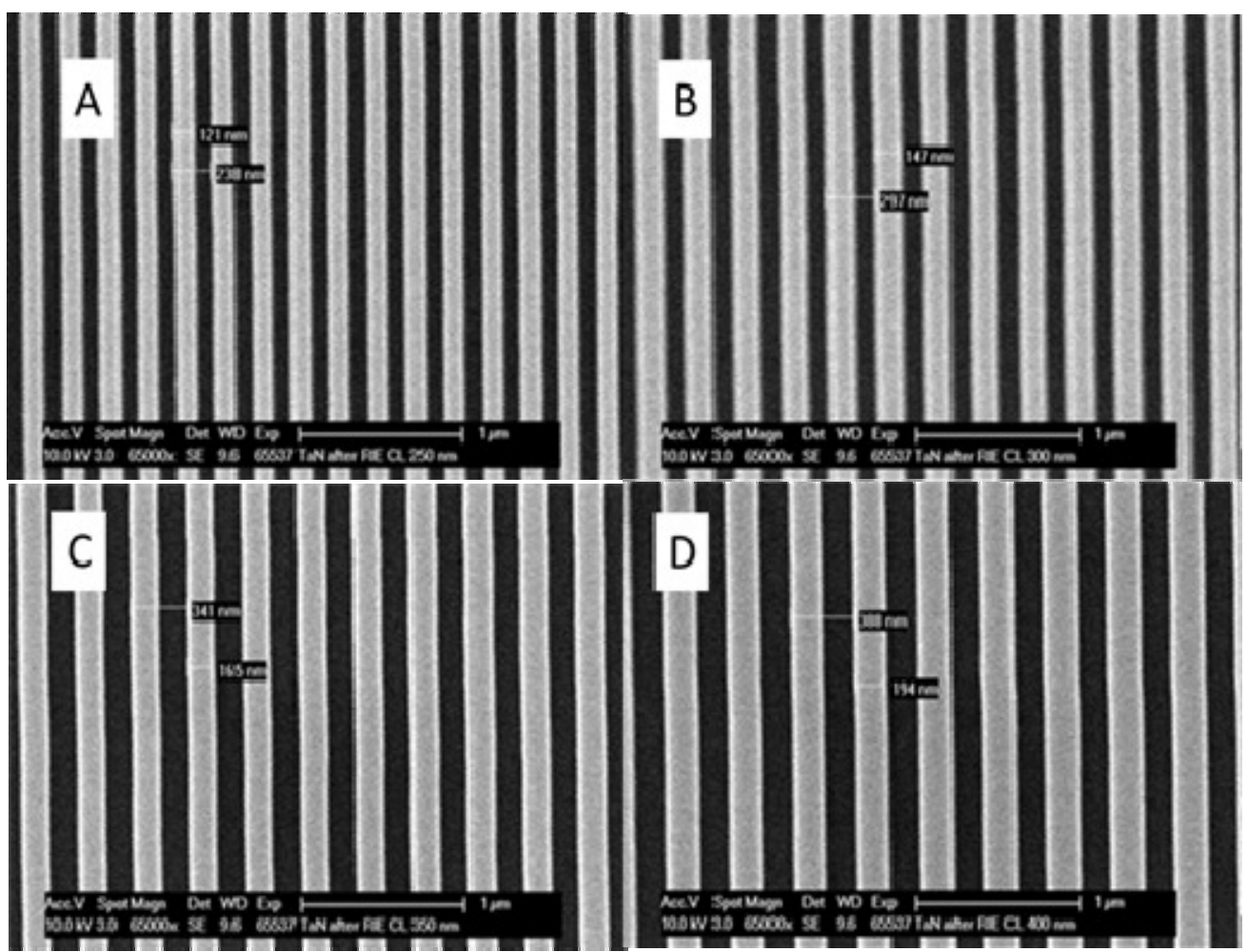

Figure 2: SEM images of the gratings after fabrication, dimensions are a) $238 \mathrm{~nm}$, b) $297 \mathrm{~nm}$, c) $341 \mathrm{~nm}$ and d) $388 \mathrm{~nm}$. 
The structures were written in the resist with the aid of an e-beam pattern generator and developed. Finally the TaN was etched with RIE Chlorine plasma and the remaining part of the resist was stripped using a RIE Oxygen plasma. SEMEDX analysis was used to verify the presence of $\mathrm{TaN}$ on lines and $\mathrm{Ru}$ in the trenches and the absence of carbon caused by possible remaining resist. SEM was also used to verify the dimensions of the gratings, see Figure 2, the measured dimensions for a line and space are: 238, 297, 341 and $388 \mathrm{~nm}$.

\subsection{Sample contamination}

After fabrication of the samples, the latter were contaminated with carbon using our carbon growth setup. This setup consists of an Ultra High Vacuum chamber equipped with a low energy e-gun from Kimball Physics and a gas supply for dodecane with a leak valve for setting pressure, see Figure 3. The samples are mounted in the vertical plane and alignment is verified using a fluorescent target. After focusing the e-gun produces a spot of about $1 \mathrm{~mm}^{2}$ at an electron energy of $1 \mathrm{keV}$. An amorphous carbon layer is formed during illumination with a back ground pressure of $1 * 10^{-6} \mathrm{mbar}$ of dodecane. The growth rate of carbon in the setup is approximately $2 \mathrm{~nm} /$ hour and was verified with interferometry. The samples were exposed for 4 hours in the system leaving a carbon layer of approximately $10 \mathrm{~nm}$. The focus does contain some hot spots and the carbon is not completely uniform. From previous experiments we have learned that if the carbon layer becomes relatively thick $(>30 \mathrm{~nm})$ the density increases in the bottom part of the layer and is harder to remove, therefore we limited ourselves to a layer of about $10 \mathrm{~nm}$. In total 3 samples were prepared with an average carbon thickness of about 10 to $25 \mathrm{~nm}$.
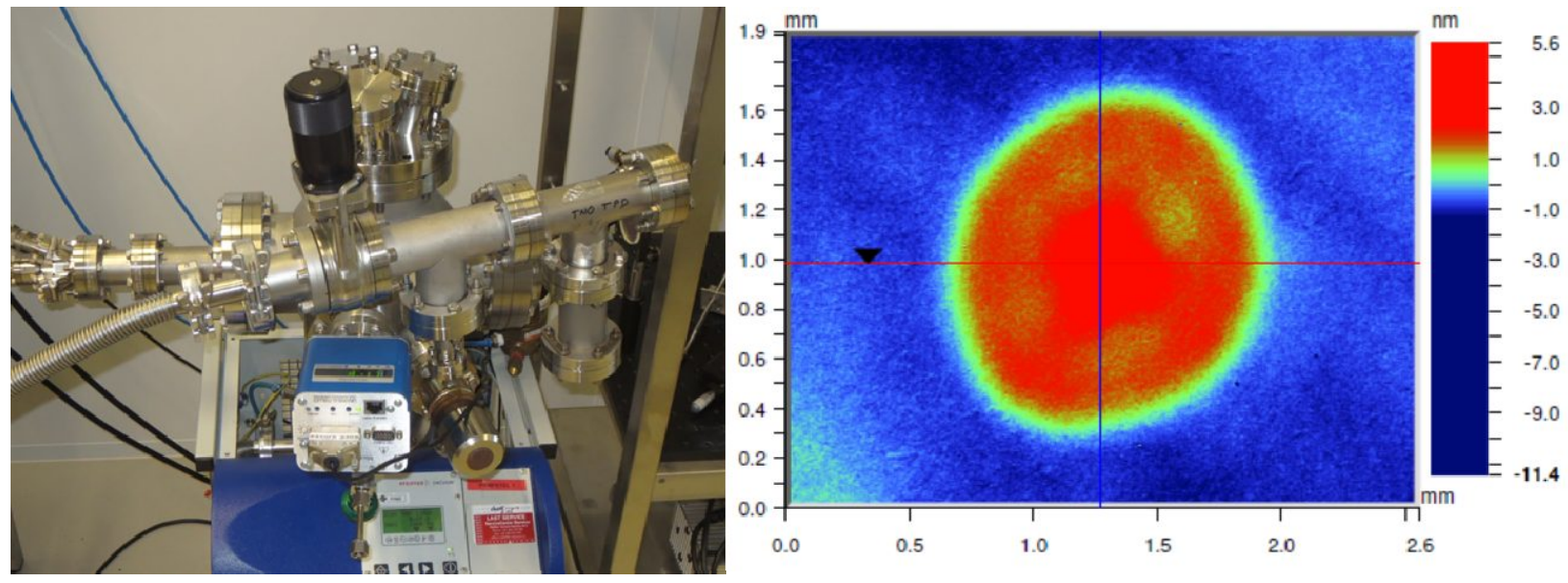

Figure 3: a) carbon growth setup with on the left side the e-gun b) typical profile of a grown carbon spot measured with interferometry.

\subsection{Sample cleaning}

After contamination with carbon two samples were cleaned to different degrees of cleanliness. For the cleaning our Plasma Analysis and Test Setup (PATS) was used, see Figure 4. The cleaning was performed using $\mathrm{H}_{2}$ as active gas, settings for the source were $1000 \mathrm{~W}$ microwave power, $50 \%$ duty cycle with on pulse of $500 \mu$ s and a pressure of 0.2 mbar of $\mathrm{H}_{2}$. Samples were mounted at a distance of $6 \mathrm{~cm}$ from the source and the cleaning runs were time maximized to 15 - $30 \mathrm{~min}$ to prevent over heating of the sample. Sample DII-3/1 was cleaned for a total time of 30 min removing about $5 \mathrm{~nm}$ of the total contamination. Sample DII-3/5 was cleaned for a total cleaning time of $120 \mathrm{~min}$, which should remove all of the contamination.

The PATS has a total of four MW sources capable of running simultaneously or in a timed pulsed mode. The last mode has the advantage of having a better penetration into small crevices and high aspect ratio trenches. For our experiments this mode was not used and only one source was used for the cleaning. The PATS has also capabilities of plasma 
analysis with a Langmuir probe, retarding field analyzer and optical emission spectroscopy. For small samples a sample holder in the center of the chamber is available and at that position the sample can be monitored using a long focal length ellipsometer.

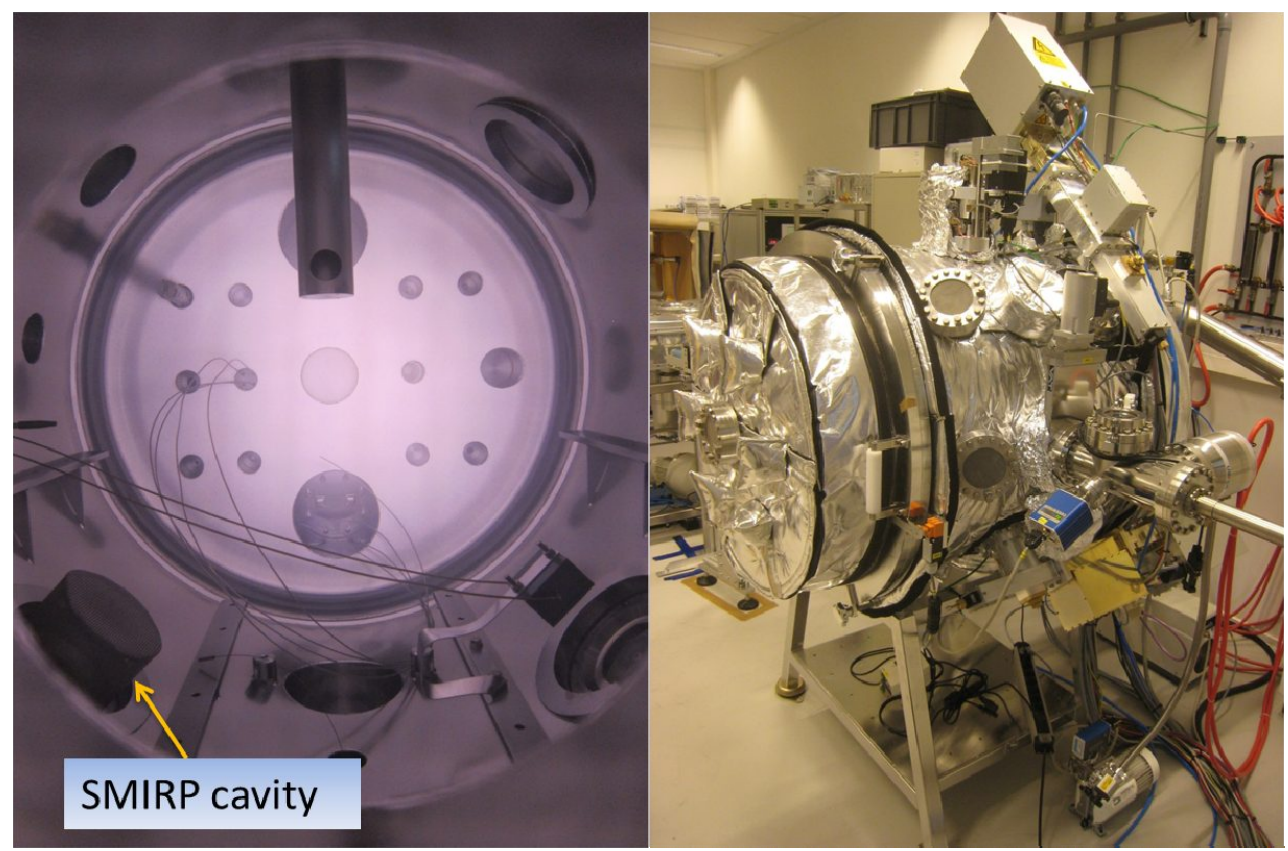

Figure 4: Plasma cleaner PATS with at the left side the interior showing a SMIRP cavity in the lower left corner.

\section{SAMPLE ANALYSIS}

In total 4 samples were send to Luxembourg for analysis: A reference sample, which had seen no contamination and no cleaning only processing for the structures. Sample DII-3/6 was contaminated with carbon but had seen no cleaning. Sample DII-3/1 had been cleaned for 30 minutes removing about $5 \mathrm{~nm}$ of the contamination and sample DII- $3 / 5$ which had been cleaned for 120 min removing about all of the carbon contamination. Sample DII-3/1 has not been analyzed, due to time constraints.

Analysis was performed using Secondary Ion Mass Spectrometry (SIMS) and Atomic Force Microscopy combined into one single system ${ }^{[3]}$. SIMS measurements were performed on a Cameca NanoSIMS 50 instrument in the negative secondary ion mode using a $16 \mathrm{keV} \mathrm{Cs}+$ primary beam. Measurements consisted of 60 or 70 consecutive scans with a dwell time of $10 \mathrm{~ms} /$ pixel respectively and a primary ion beam current on the sample of $0.5 \mathrm{pA}$. The recorded secondary ion signals were 16O-, 12C-12C2- and 28Si-. For the AFM analysis High Aspect Ratio tips with a spike length of $2 \mu \mathrm{m}$ (HARTA0-2) from AppNano were used.

In Figure 5 the AFM line scans are shown for the 3 samples (ref, contaminated and fully cleaned). The scans are taken on the structure with $300 \mathrm{~nm}$ lines and spaces. From the scan of the reference sample it can be observed that the bottom of the trench is not fully resolved by the AFM tip and that the height of the TaN layer is at least $50 \mathrm{~nm}$ and probably more. The line scan of sample DII-3/6 (CNC in figure) shows that the depth of the trenches has been greatly reduced to about $25 \mathrm{~nm}$ and that the top of the line has an added sharp feature. This indicates that the carbon layer has a much greater height than anticipated and that the trench is almost fully filled with carbon. The line scan of sample DII-3/5, which had been cleaned for full carbon removal, shows that the depth of the trench has been restored to at least $50 \mathrm{~nm}$. The top of the line still shows a remainder of the features that were clearly visible in the carbon coated sample. This again indicates that not all carbon was removed and that the layer is thicker than anticipated. 


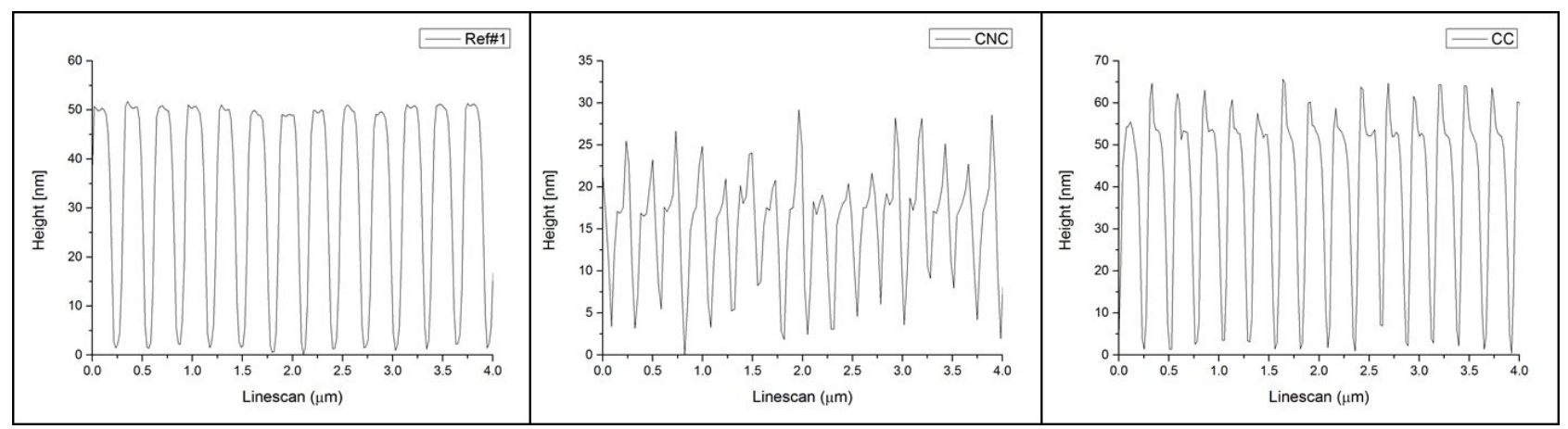

Figure 5: AFM line scans of the $300 \mathrm{~nm}$ lines and spacing structure of the reference sample, carbon coated sample (CNC) and fully cleaned sample (CC).

Figure 6 shows the sputter depth profiles of the three analyzed samples. On all samples the depth profile was determined on top of the line (ridge) and in the bottom of the trench. For each profile several slices were executed until the Si wafer substrate was reached. This takes typically 30 slices before the Si signal stabilizes and all the $\mathrm{C}, \mathrm{TaN}$ and Ru has been sputtered away.
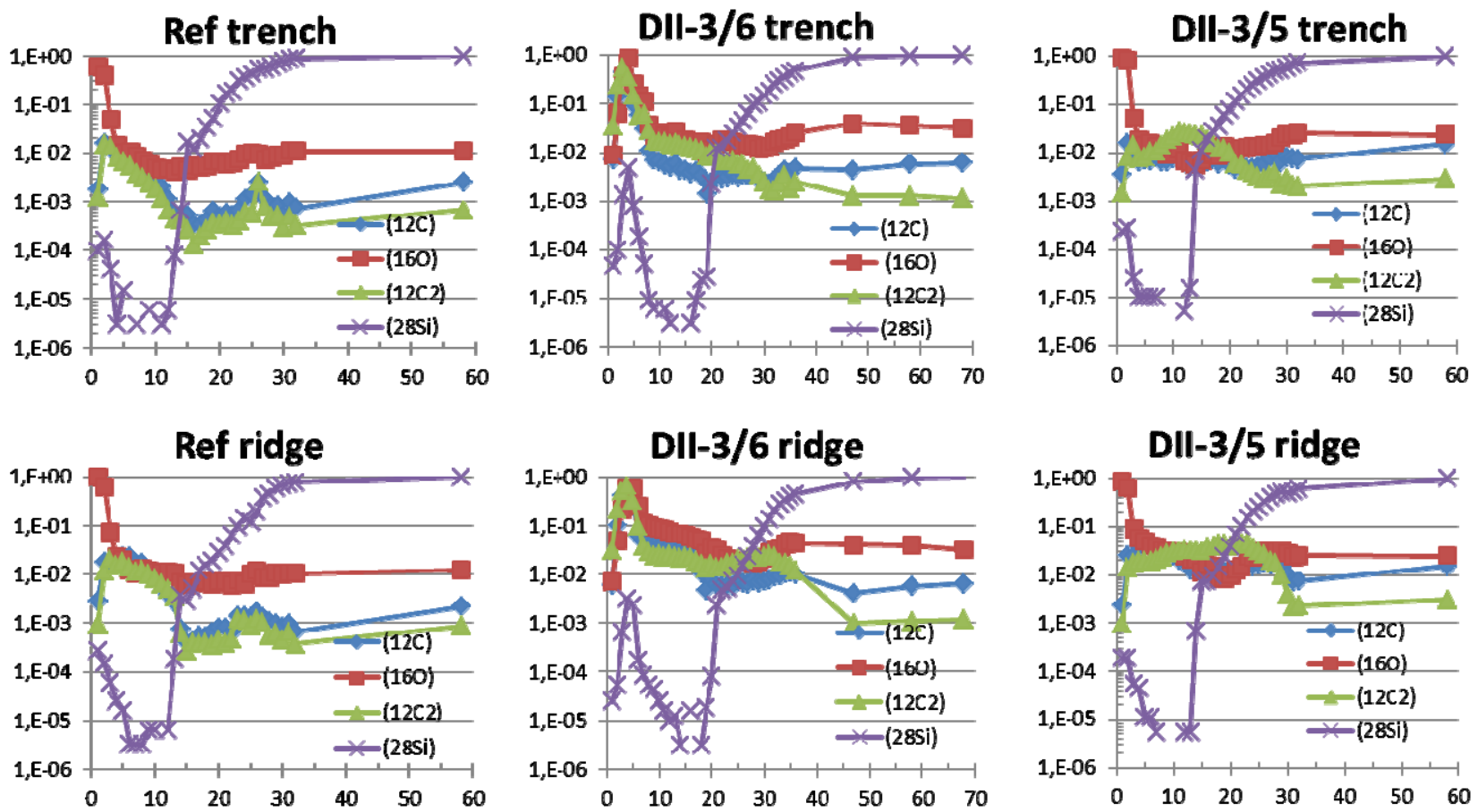

Figure 6: SIMS depth profiles of the reference sample (Ref), the contaminated sample (DII-3/6) and the cleaned sample (DII-3/5) for the trench and the lines (ridge). In all figures, the detected secondary ion signals were normalized to the average $\mathrm{Si}$ - bulk signal. The detection of the TaN- signal was only limited and is not shown. The horizontal axis is the number of the raster slice.

The reference sample shows some oxygen on top of the surface, both in the trench as in the ridge, it takes about 12 slices before the Si starts to show up in the SIMS spectra and with 30 slices the Si starts to peak indicating that the wafer has been reached by the sputter gun. The contaminated sample clearly shows carbon on top of the surface and it takes about 6 slices to cut through the carbon layer. It is interesting to see that the Si signal of the contaminated sample spikes together with the carbon peak. The completely cleaned sample shows a restored spectrum when compared to the reference, with a similar number of slices needed to reach the wafer. Also the oxygen peak on the surface has been restored and the Si peak which is visible in the contaminated sample in the first slices has disappeared. The carbon signal 
in the trench of the cleaned sample however continuous for much more slices. This could be an indication that a small amount of carbon may still be present as also could be concluded from the AFM scans. The amount of carbon however is still very small.

\section{CONCLUSION}

In this experimental work we have shown that it is possible to remove carbon inside the trenches of a EUV reticle, although a very small amount of carbon may still be present. This could be caused by a much thicker edge layer of carbon to begin with or by contamination during transport. The signal for oxygen and $\mathrm{Si}$ was restored to its original shape and value. This technique using plasma with $\mathrm{H}_{2}$ as active species seems to be a potential candidate for cleaning of reticles even with the pellicle still attached. The SIMS-SPM system is a powerful tool for verifying the presence of carbon in trenches in combination with the AFM images also dimensional changes can be monitored.

\section{ACKNOWLEDGEMENTS}

We wish to thank Hans van den Berg for preparing the samples and doing the SEM-EDX analysis. This work was made possible by funding of the Dutch national NanoNext program.

\section{REFERENCES}

[1] L. Scaccabarozzi, N.A. Lammers, R. Moors, and V. Banine, Particle Cleaning of EUV Reticles, Journal of Adhesion Science \& Technology, 23 (2009) 1603-1622.

[2] N.B. Koster et al, "New developments in cleaning of EUVL mirrors and reticles", EUVL symposium 2009, Praque, http://www.sematech.org/meetings/archives/litho/8653/pres/O_RC-03_Koster_TNO.pdf

[3] T. Wirtz, Y. Fleming, M. Gerard, U. Gysin, T. Glatzel, E. Meyer, U. Wegmann, U. Maier, A.H. Odriozola, and D. Uehli, Design and performance of a combined secondary ion mass spectrometry-scanning probe microscopy instrument for high sensitivity and high-resolution elemental three-dimensional analysis, Review of Scientific Instruments, 83 (2012) 063702-063709. 